\title{
Plasma Waves in the Sun
}

\author{
Vipin K. Yadav \\ Space Physics Laboratory, Vikram Sarabhai Space Centre, Thiruvananthapuram 695 022, Kerala, India
}

Copyright $\subseteq 2016$ by authors, all rights reserved. Authors agree that this article remains permanently open access under the terms of the Creative Commons Attribution License 4.0 International License

\begin{abstract}
Plasma waves are observed in almost all the solar system objects such as planets, their satellites, comets, interplanetary medium (IPM) and Sun. In most of these solar system plasma environments, the typical plasma density has a range between $10^{3}-10^{6} \mathrm{~cm}^{-3}$ and plasma temperature of about $0.1 \mathrm{eV}$ which is capable of sustaining some plasma waves of the complete wave spectrum. In IPM, some natural plasma modes are observed whose origin is believed to be near the Sun. The plasma in solar core and in corona itself support and sustain a number of electrostatic (ES) and electromagnetic (EM) plasma waves. The solar plasma waves carry energy away from the Sun to far-off places as well as incite particle transport from the solar core to interplanetary space. These waves also provide information on energy distribution in solar plasma. This paper revisits the plasma waves coming from Sun towards Earth in the form of a brief review.
\end{abstract}

Keywords Electrostatic and Electromagnetic Plasma Waves, Solar Plasma, Interplanetary Medium, Langmuir Waves, Ion-acoustic Waves, Whistler Waves, Alfven Waves

\section{Introduction}

Plasma waves are omnipresent in space plasmas and are observed in our solar system $[1,2]$. They propagate energy across different space regions, transport particles in the absence of collisions, and provide acceleration to particles to attain high energies. They also have an imprint of information about the local plasma properties and thus are useful to derive information from regions not accessible for in-situ measurements such as near the Sun. Due to the high temperatures in the solar corona, local direct measurements there are extremely difficult. Moreover, the plasma waves that come out from Sun or evolve near the Sun carry the history along with them. By history, it is meant here that from which physical process the wavers get generated. There exist about 50 different plasma instabilities [3] which grow on encountering the favorable conditions of non-linear energy build-up in various plasma systems. Each one of these system relaxes by releasing out the excess energy in the form of plasma waves. These plasma waves are specific to different instabilities and their properties depend upon the background plasma environment prevailing there at that location.

This paper is organized as follows: In section 2, a brief description of solar plasma environment is given. The earlier missions, sent so far to study the solar plasma and waves are described in section 3 . In section 4 , various plasma waves generated in and around the Sun are compiled. Section 5 comprises of the possible plasma waves in the solar wind. Some of the unresolved solar plasma mysteries are presented in section 6. Section 7 provides the overall conclusion.

\section{Solar Plasma Environment}

In Sun, there is a clear distinction between the magnetic fields and plasmas in the solar core, in photosphere and that in corona. In the core of Sun, both plasma density and plasma temperature are very high. In photosphere, the plasma density is very high when compared with the corona. On the other hand, the plasma temperature in photosphere is about three orders less than that exist in corona. These three solar regions are shown in Figure 1.

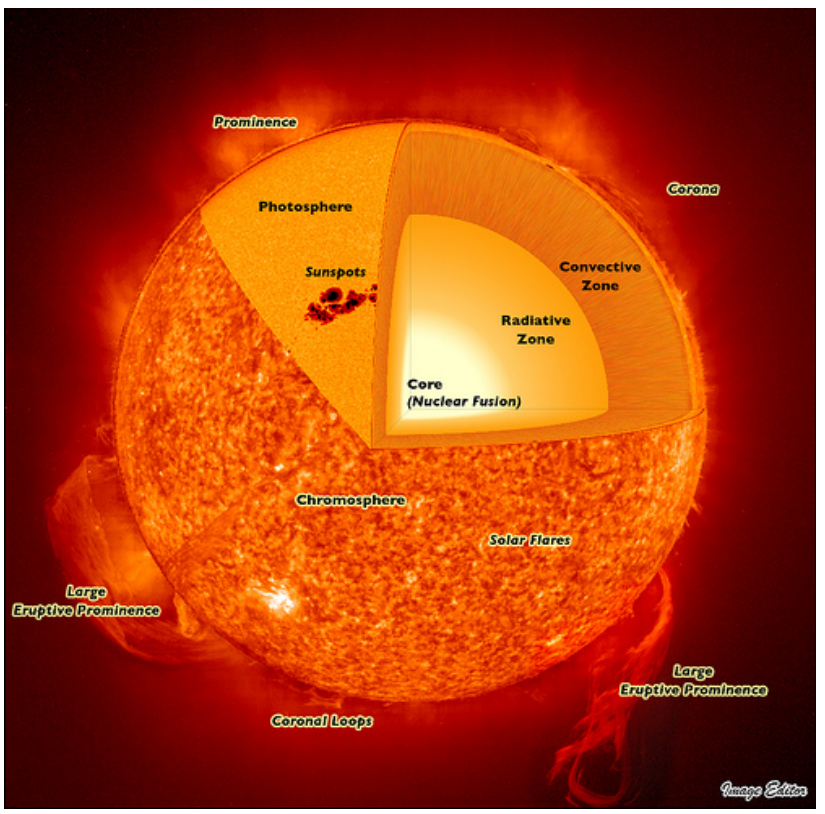

Figure 1. The interior of Sun indicating various zones and main features [image courtesy: www.ualberta.ca]. 
The primary plasma parameters - density and temperature and the magnetic field in the three regions of Sun are given in Table 1.

Table 1. Plasma parameters and magnetic field in Sun [4]

\begin{tabular}{|c|c|c|c|c|}
\hline $\begin{array}{c}\text { S. } \\
\text { No. }\end{array}$ & Parameter & Core & Photosphere & Corona \\
\hline 1. & $\begin{array}{c}\text { Plasma density, } \\
\mathrm{n}_{\mathrm{e}}\left(\mathrm{cm}^{-3}\right)\end{array}$ & $1 \times 10^{26}$ & $8 \times 10^{16}$ & $1 \times 10^{9}$ \\
\hline 2. & $\begin{array}{c}\text { Temperature, T } \\
(\mathrm{K})\end{array}$ & $1.5 \times 10^{7}$ & $6 \times 10^{3}$ & $2 \times 10^{6}$ \\
\hline 3. & $\begin{array}{c}\text { Magnetic Field, } \\
\mathrm{B}(\mathrm{T})\end{array}$ & --- & $0.1-0.3$ & 0.01 \\
\hline
\end{tabular}

\section{Missions to study the solar plasma waves}

Till now there have been sixteen missions namely Helios 1 \& 2, ISEE-3, Ulysses, YOHKOH, WIND, SOHO, ACE, TRACE, GENESIS, RHESSI, HINODE, STEREO, SMO, PICARD, SDO and IRIS by various space agencies (NASA, ESA and JAXA) around the world to observe the Sun and to study the solar wind in detail. The main instruments in these spacecrafts were particle (electron, proton, and ion) detectors but six of these missions carried instruments to study solar plasma waves which are as follows:

(1) Helios 1 \& 2 were launched by NASA in collaboration with then West Germany on December 10, 1974 \& January 15, 1976 respectively. Both the spacecrafts carried identical instruments two flux-gate magnetometers, one search-coil magnetometer, one plasma wave investigation, one electric field experiment and one radio wave experiment.

(2) ISEE-3 (international Sun Earth Explorer 3) was launched by NASA/ESA on August 12, 1978, later also known as ICE (International Cometary Explorer). It was a heliocentric spacecraft which operated at about 1.5 million $\mathrm{km}$ from the Earth towards Sun. The scientific aims of this mission were to study the solar wind plasma at a place distant from that of Earth's magnetosphere and to investigate the solar wind composition in the interplanetary region at a distance of $\sim 1 \mathrm{AU}$ from Sun. It carried ISEE-C plasma wave instrument which comprised of a long body-mounted electric dipole ( $90 \mathrm{~m}$, tip-to-tip), a short boom-mounted electric dipole $(0.6 \mathrm{~m}$, effective length) [Frequency range: $17 \mathrm{~Hz}-100 \mathrm{kHz}, 17 \mathrm{~Hz}-1 \mathrm{kHZ}, 0.316 \mathrm{~Hz}-$ $8.8 \mathrm{~Hz}]$ and a high-sensitivity search-coil magnetometer [5].

(3) Ulysses was launched on October 6, 1990 by NASA and ESA in collaboration to study the Sun at all latitudes. Apart from a particle detector SWOOPS designed to measure the electrons and positive ions and a solid state $\mathrm{x}$-ray instrument to study $\mathrm{x}$-rays from solar flares, two plasma wave detection instruments were also there onboard Ulysses -

i. Two Radio/Plasma antennas in dipole mode and one in monopole mode used to measure the radio waves generated by plasma in the frequency range of $\mathrm{DC}$ to $1 \mathrm{MHz}$;

ii. Two magnetometers - one vector helium \& one fluxgate to measure the background magnetic field and a two-axis search-coil magnetic antenna to measure the time varying magnetic fields.

(4) WIND, a spin stabilized spacecraft was launched by NASA on November 1, 1994. The main scientific objective of WIND was to measure the solar wind properties before it reaches the Earth thereby investigating the basic plasma processes near the Earth and to provide complete plasma, energetic particles and magnetic field information. WIND has two main instruments for plasma wave studies -

i. Magnetic Field Investigation (MFI) having two tri-axial fluxgate magnetometers (Dynamic range: $4 \mathrm{nT}-65.5 \mu \mathrm{T}$ ) to measure the steady state background magnetic field [6];

ii. Radio and Plasma Investigation (WAVES) designed to measure the intensity and arrival direction for both propagating and in-situ waves originating in the solar wind near the Earth. It has a total of six antenna units in the frequency range of a fraction of $\mathrm{Hz}-14 \mathrm{MHz}$ for the electric field measurements and three magnetic search coils mounted orthogonally to each other up to $3 \mathrm{kHz}$ frequency [7].

(5) ACE (Advanced Composition Explorer) was launched by NASA on August 25, 1997 to study the energetic particles from the solar wind and interplanetary medium at L1 point. ACE had nine instruments onboard to detect solar wind particles and one magnetometer (MAG) to measure the background interplanetary magnetic field [8].

(6) STEREO (Solar Terrestrial RElations Observatory) was launched by NASA on October 26, 2006. It has two spacecrafts A \& B which were ejected in the opposite directions so that with time the two spacecrafts would continue to separate from each other at a combined rate of $\sim 44^{\circ}$ per year. A \& B achieved $90^{\circ}$ separation in January 2009 and $180^{\circ}$ in February 2011 which provided an opportunity to observe the complete Sun for the first time. Two main plasma instruments onboard STEREO -

i. PLAsma and SupraThermal Ion Composition (PLASTIC) to study the plasma properties of protons, $\alpha$ particles and heavy ions;

ii. STEREO/WAVES (S/WAVES) instrument, a radio burst tracker to study radio 
disturbances from Sun to Earth, has three orthogonal monopole antennas having a length of $6 \mathrm{~m}$ mounted on the back (anti-sunward) surface of the spacecraft. The low frequency receivers (LFR) were in 3 bands: A $(2.5-10 \mathrm{kHz}), \mathrm{B}(10-40 \mathrm{kHz})$ and C $(40-160 \mathrm{kHz})$. The high frequency receiver (HFR) was a sweeping signal receiver operating in the frequency range $125 \mathrm{kHz}-16.025 \mathrm{MHz}$ [9].

The best place to detect and study the solar plasma waves are the Lagrangian points particularly the first Lagrangian (L-1) point which is located at a distance of about $1.5 \times 10^{6}$ $\mathrm{km}$ from the Earth towards the Sun on a line joining the centres of these two solar system objects. Any spacecraft positioned at this location has a stable orbit and is sufficiently away from the Earth's magnetosphere to make pristine measurements.

\section{Plasma Wave Generation near the Sun}

It is reported that the plasma instabilities occurring in the vicinity of Sun generate a number of plasma waves of different modes [10]. Energetic electrons beams come out from the core of sun and encounter a Maxwellian plasma distribution in the corona. Due to resonant damping, this electron beam is absorbed in the energetic tail of the coronal plasma and due to this a non-linear energy build up takes place there which results in triggering bump-on-tail instability [11]. Thus, electrostatic Langmuir waves are produced and propagate in the extended solar corona up to large distances. These Langmuir or electron plasma waves cannot come out of the plasma as electrostatic hence, they got mode converted into electromagnetic waves and leave the plasma for interplanetary medium to reach the L-1 point.

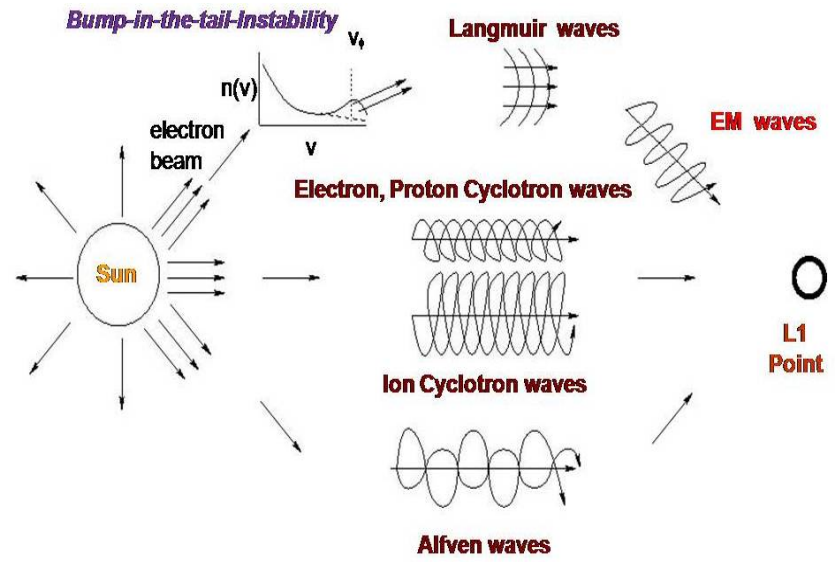

Figure 2. Schematic of the various plasma wave generations and their subsequent propagation towards Earth

Electromagnetic Ion cyclotron waves are predicted to emanate from the solar corona along with the Coronal Mass
Ejection (CME) due to the kinetic instability driven by the anisotropy in the ion temperature [12]. This kinetic instability gets saturated in the non-linear stage due to the wave particle interactions resulting in the modification of ion distribution to reduce the temperature anisotropy and to excite the waves. The schematic of some of these plasma wave generations in the solar environment and their propagation is depicted in Figure 2.

The heat flux in a collisionless plasma by electrons attempts to distort the distribution function in such a way that the perpendicular averaged velocity distribution in parallel direction has a tail in the form of a beam which is unstable and grows in the form of an instability called electron-heat-flux instability thereby exciting collective modes such as electromagnetic whistler waves [13]. The ion acoustic waves (IAW) observed in the solar wind at frequencies between ion and electron plasma frequencies $\left(\mathrm{f}_{\mathrm{pi}}\right.$ $\left.<\mathrm{f}<\mathrm{f}_{\mathrm{pe}}\right)$ is closely correlated with the electron to proton temperature ratio $\left(\mathrm{T}_{\mathrm{e}} / \mathrm{T}_{\mathrm{p}}\right)$ and with the electron heat flux [11]. Plasma waves are also generated by the thermal and non-thermal particle distributions of the plasma populations such as the electron and proton cyclotron waves [1].

Although it is known that Alfven waves gets generated in the plasma of solar corona, the observation there gets hindered due to the incompressible nature of Alfven waves which makes them invisible as intensity fluctuations so that the intensity imagers for coronal observations could not observe them [13]. Also, most of the solar spectrographs employed had a limited field of view in the set time interval which is insufficient and short compared to the wave periods so as to make a meaningful observation of the velocity fluctuation from the Doppler shifts of emission lines [14]. The discontinuities in the solar magnetic field give rise to plasma disturbances namely the Alfven waves which have two modes - the fast mode and the slow mode. The fast mode Alfven wave gets absorbed in the solar corona but the slow mode Alfven travels a long distance away from the Sun in the interplanetary medium [15].

Table 2. Various plasma instabilities and their possible causes in solar plasma environment [16]

\begin{tabular}{|c|c|c|c|}
\hline $\begin{array}{c}\text { S. } \\
\text { No. }\end{array}$ & Frequency & Natural mode & Possible cause \\
\hline 1. & $\mathrm{f} \sim \mathrm{f}_{\mathrm{pe}}$ & Langmuir waves & $\begin{array}{c}\text { Electron-beam } \\
\text { instability }\end{array}$ \\
\hline 2. & $\mathrm{f}<\mathrm{f}_{\mathrm{pi}}$ & Ion acoustic waves & Ion-beam instability \\
\hline 3. & $\mathrm{f}<\mathrm{f}_{\mathrm{pe}}$ & $\begin{array}{c}\text { Electron acoustic } \\
\text { waves }\end{array}$ & $\begin{array}{c}\text { Electron-beam } \\
\text { instability }\end{array}$ \\
\hline 4. & $\mathrm{f}<\mathrm{f}_{\mathrm{ce}}$ & $\begin{array}{c}\text { Whistler/Lower } \\
\text { hybrid waves }\end{array}$ & $\begin{array}{c}\text { Electron-heat-flux } \\
\text { instability }\end{array}$ \\
\hline
\end{tabular}

Various natural plasma frequencies in solar plasma can excite different plasma waves depending upon the prevailing conditions there [16]. These possible plasma instabilities arising due to comparative natural frequencies in the solar plasma environment are tabulated in the Table 2. 


\section{Plasma Wave in the Solar Wind}

Ion Acoustic waves are generated in the solar wind due to electron-heat-flux instability and the double-ion-beam instability [17]. The electron-heat-flux caused asymmetry in the electron velocity distribution function which worked as the free energy source for this instability to grow. On the other hand, the occurrence of double-proton streams in the proton distribution function acts as the free energy source for the double-ion-beam instability [17].

WIND spacecraft, in June 2007, observed pulse-like Alfvenic disturbances in the solar wind which were isolated events characterized by nearly plane-polarized rotations in the solar wind magnetic field and velocity vectors away from the directions of the background field and velocity and then back again [18]. The observations are shown in Figure 3.

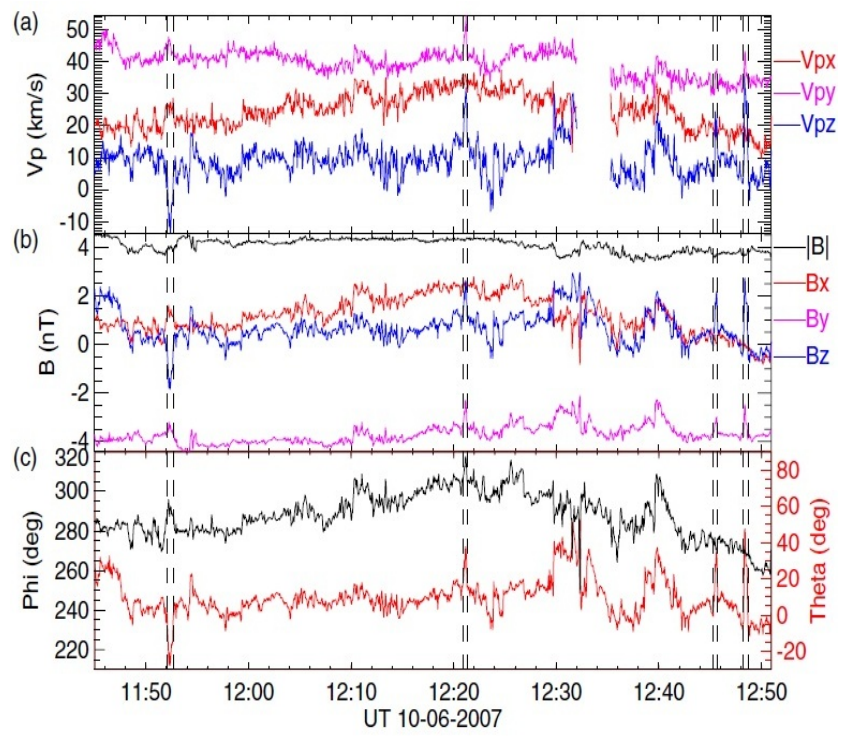

Figure 3. Alfven waves observed in the solar wind by the WIND [18]

\section{Solar Plasma Phenomena and the Unresolved Issues}

Despite the fact that there were about six missions dedicated to study solar plasma and the associated phenomena such as plasma waves and magnetic disturbances, the following scientific issues are only partially addressed and are there still unresolved [5]:

1) The role that plasma waves play at interplanetary discontinuities and at stream-stream interaction fronts as when there is a discontinuity, some energy has to propagate away from there which provide a non-local wave-particle interaction mechanism.

2) The analysis of the basic interplanetary instabilities associated with thermal anisotropy and heat conduction that cause the solar wind to behave as a fluid even when the mean free path for solar wind particles is very large at a distance of about $1 \mathrm{AU}$ from the Sun.
3) The complete understanding of the localized wave-particle acceleration processes in the solar wind and the transport associated with wave-particle interaction processes in the solar wind at a distance away from the Sun.

4) The insight of energy loss and wave-wave conversion mechanisms for highly energetic electrons and protons by correlating particle distribution data with wave measurements.

5) The possible local plasma parameter estimation such as solar plasma density and solar plasma temperature using data from the plasma waves coming from the Sun.

6) The interplanetary whistler-mode signals that can develop when there is an-isotropy in the electron temperature (when $\mathrm{T}_{\perp} / \mathrm{T}_{\|}>1$ ).

7) The understanding of dynamical energy dissipation processes that can cause large amplitude magnetohydrodynamic (MHD) waves in the solar wind to steepen into collisionless shocks.

In this scenario, it shall be prudent to send a plasma wave detection instrument on any future solar mission to improve our understanding of plasma waves generated near the Sun and coming towards the earth.

\section{Conclusions}

The Sun is a constant source of plasma waves. However, these waves cannot be detected by making in-situ measurements near the Sun because of the prevailing extreme thermal conditions existing there. Hence, the observations are made at a place far-off from the Sun where a number of plasma waves are observed, believed to be originated from the Sun. Some plasma waves are observed in the interplanetary medium also the origin of which can be linked to the Sun. The primary sources of generation of these waves are various instabilities that take place in and near the Sun due to its solar dynamics. A number of missions are sent towards the Sun to study it but the missions having plasma wave detection instruments onboard were very few. There are still a large number of unresolved scientific issues associated with solar plasma and waves that are required to be addressed in the future missions to study the Sun. Hence, it is must to have a suit of plasma wave detection instruments onboard any future space mission to study the Sun.

\section{REFERENCES}

[1] Vipin K. Yadav, Anil Bhardwaj. "Plasma Waves in Planetary Ionosphere-Magnetosphere System", Conference on Planetary Science and Exploration, Ahmedabad, India, December 12-14, 2011; 160-161, 2011. 
[2] Vipin K. Yadav, R. S. Thampi, Anil Bhardwaj. "Plasma Waves in the Solar System", $27^{\text {th }}$ National Symposium on Plasma Science and Technology (Plasma-2012), Pondicherry University, Pudducherry, India. December 10-13, 2012, 454-458, 2013

[3] Akira Hasegawa, Plasma Instabilities and Non-linear Effects, Springer-Verlag, New York, Heidelberg, Berlin, 1975.

[4] Karl Schnider, Physics of Space Plasma Activity, Cambridge University Press, UK, 2007.

[5] F. L. Scarf, R. W. Fredricks, D. A. Gurnett, E. J. Smith, "The ISEE-C Plasma Wave Investigation", IEEE Transactions on Geoscience Electronics, Vol. GE-16, No. 3, 191-195, 1978.

[6] R. P. Lepping, M. H. Acuna, L. F. Burlaga, W. M. Farrell, J. A. Slavin, K. H. Schatten, F. Mariani, N. F. Ness, F. M. Neubauer, Y. C. Whang, J. B. Byrnes, R. S. Kennon, P. V. Panetta, J. Scheifele, E. M. Worley, "The WIND Magnetic Field Investigation", Space Science Reviews, Vol. 71, 207-229, 1995.

[7] J.-L. Bougeret, M.L. Kaiser, P.J. Kellogg, R. Manning, K. Goetz, S.J. Monson, N. Monge, L. Friel, C.A. Meetre, C. Perche, L. Sitruk, S. Hoang, "WAVES: The Radio and Plasma Wave Investigation of the WIND Spacecraft", Space Science Reviews, Vol. 71, 231-263, 1995.

[8] C.W. Smith, J. L'Heureux, N.F. Ness, M.H. Acuna, L.F. Burlaga, J. Scheifele, "The ACE Magnetic Field Experiment", Space Science Reviews, Vol. 86, 613-632, 1998.

[9] J.-L. Bougeret, K. Goetz, M. L. Kaiser, S. D. Bale, P. J. Kellogg, M. Maksimovic, N. Monge, S. J. Monson, P. L. Astier, S. Davy, M. Dekkali, J. J. Hinze, R. E. Manning, E. Aguilar-Rodriguez, X. Bonnin, C. Briand, I. H. Cairns, C. A. Cattell, B. Cecconi, J. Eastwood, R. E. Ergun, J. Fainberg, S. Hoang, K. E. J. Huttunen, S. Krucker, A. Lecacheux, R. J. MacDowall, W. Macher, A. Mangeney, C. A. Meetre, X. Moussas, Q. N. Nguyen, T. H. Oswald, M. Pulupa, M. J. Reiner, P. A. Robinson, H. Rucker, C. Salem, O. Santolik, J.
M. Silvis, R. Ullrich, P. Zarka, I. Zouganelis, Space Science Reviews, Vol. 136, 487-528, 2008.

[10] K. Papadopoulos, Lecture Notes Physics, Chapter 1: Waves \& Instabilities in Space Plasmas, Vol. 778, 1-43, Springer-Verlag Berlin Heidelberg, 2009.

[11] O. Moullard, D. Burgess and S. D. Bale, "Whistler waves observed during an in-situ solar type III radio burst", Astronomy \& Astrophysics, Vol. 335, 703-708, 1998.

[12] C. J. Farrugia, F. T. Gratton, G. Gnavi, K. W. Ogilvie, “On the possible excitation of electromagnetic ion cyclotron waves in solar ejecta", Journal of Geophysical Research, Vol. 103, No. A4, 6543-6550, 1998 .

[13] S. Peter Gary, W. C. Feldman, D. W. Forslund, M. D. Montgomery, "Electron Heat Flux Instabilities in the Solar Wind", Geophysical Research Letters, Vol. 2, No. 3, 79-82, 1975.

[14] S. Tomczyk, S. W. McIntosh, S. L. Keil, P. G. Judge, T. Schad, D.H. Seeley, J. Edmondson, "Alfven Waves in the Solar Corona", Science, Vol. 317, 1192-1196, 2007.

[15] J. W. Belcher, Leverett Davis Jr. "Large Amplitude Alfven Waves in the Interplanetary Medium 2", Journal of Geophysical Research, Vol. 76, No. 16, 3534-3563, 1971.

[16] R. J. MacDowall, R. A. Hess, N. Lin, G. Thejappa, A. Balogh, J.L. Phillips, "Ulysses spacecraft observations of radio and plasma waves: 1991-1995", Astronomy and Astrophysics, Vol. 316, 396-405, 1996.

[17] D. A. Gurnett, E. Marsch, W. Pillip, R. Schwenn, H. Rosenbauer, "Ion Acoustic Waves and related Plasma Observations in the Solar Wind", Journal of Geophysical Research, Vol. 84, No. A5, 2029-2038, 1979.

[18] J. T. Gosling, H. Tian, T. D. Phan, "Pulsed Alfven Waves in the Solar Wind", The Astrophysical Journal Letters, Vol. 737, L35, 2011. 